\title{
GSK-3 Inhibitor LY2090314
}

National Cancer Institute

\section{Source}

National Cancer Institute. GSK-3 Inhibitor LY2090314. NCI Thesaurus. Code C116849.

An inhibitor of glycogen synthase kinase-3 (GSK-3), with potential antineoplastic activity. Upon administration, LY2090314 binds to and inhibits GSK-3 in an ATP-competitive manner. This prevents GSK-3-mediated phosphorylation of beta-catenin, which inhibits the subsequent ubiquitination and proteasomal degradation of beta-catenin. This leads to the activation of the Wnt/beta-catenin pathway and the induction of apoptosis in susceptible tumor cells. GSK-3, a serine/threonine kinase, plays a key role in numerous pathways involved in protein synthesis, cellular proliferation, differentiation, and apoptosis. The Wnt/beta-catenin signaling pathway plays key roles in both cellular proliferation and differentiation. The increased expression of beta-catenin, a transcriptional activator, correlates with decreased cellular proliferation and improved prognosis in select cancers. 